\title{
Preterm Children Have Disturbances of White Matter at 11 Years of Age as Shown by Diffusion Tensor Imaging
}

\author{
ZOLTAN NAGY, HELENA WESTERBERG, STEFAN SKARE, JESPER L. ANDERSSON, \\ ANDERS LILJA, OLOF FLODMARK, ELISABETH FERNELL, KIRSTEN HOLMBERG, \\ BIRGITTA BÖHM, HANS FORSSBERG, HUGO LAGERCRANTZ, AND TORKEL KLINGBERG \\ Neonatal Unit [Z.N., H.L.], Neuropediatric Unit [Z.N., H.W., E.F., K.H., B.B., H.F., T.K.] of the \\ Department of Women and Child Health at the Astrid Lindgren Children's Hospital, Department of \\ Neuroradiology [A.L., O.F.], and Department of Clinical Neuroscience, MR Center [S.S., J.L.A.],
} Karolinska Institutet, Q2:07, 17176 Stockholm, Sweden

\begin{abstract}
Preterm birth frequently involves white matter injury and affects long-term neurologic and cognitive outcomes. Diffusion tensor imaging has been used to show that the white matter microstructure of newborn, preterm children is compromised in a regionally specific manner. However, until now it was not clear whether these lesions would persist and be detectible on longterm follow-up. Hence, we collected diffusion tensor imaging data on a 1.5-T scanner, and computed fractional anisotropy and coherence measures to compare the white matter integrity of children born preterm to that of control subjects. The subjects for the preterm group $(10.9 \pm 0.29 \mathrm{y} ; n=9$; birth weight $\leq 1500 \mathrm{~g}$; mean gestational age, $28.6 \pm 1.05 \mathrm{wk})$ possessed attention deficits, a common problem in preterms. They were compared with age- and sex-matched control children $(10.8 \pm 0.33 \mathrm{y} ; n=$ 10 ; birth weight $\geq 2500$; gestational age, $\geq 37 \mathrm{wk}$ ). We found that the preterm group had lower fractional anisotropy values in the posterior corpus callosum and bilaterally in the internal
\end{abstract}

\section{ABSTRACT}

capsules. In the posterior corpus callosum this difference in fractional anisotropy values may partially be related to a difference in white matter volume between the groups. An analysis of the coherence measure failed to indicate a group difference in the axonal organization. These results are in agreement with previous diffusion tensor imaging findings in newborn preterm children, and indicate that ex-preterm children with attention deficits have white matter disturbances that are not compensated for or repaired before 11 y of age. (Pediatr Res 54: 672-679, 2003)
DTI, diffusion tensor imaging
FA, fractional anisotropy
IVH, intraventricular hemorrhage
MRI, magnetic resonance imaging
PVL, periventricular leukomalacia

Preterm birth, and the early exposure to the extrauterine environment, increases the risks of perinatal brain injury $(1,2)$. Often these injuries involve the white matter $(2,3)$ and affect long-term neurologic and cognitive outcome, including problems with attention (4-8). These long-term effects of perinatal complications are either a result of the inability to repair the original lesions, such as PVL, or caused by a disruption of the maturational processes.

DTI is a relatively recent method of MRI. From the fact that the preferential direction of water diffusion within the white

Received January 14, 2003; accepted April 18, 2003.

Correspondence: Zoltan Nagy, Karolinska Institutet, Department of Woman and Child Health, Neonatology Astrid Lindgrens Barnsjukhus Q2:07 17176 Stockholm, Sweden; e-mail: zoltan.nagy@kbh.ki.se

Supported by grants from the Swedish Medical Research Council 5234, the Vardal foundation, and Sällskapet Barnavård.

DOI: 10.1203/01.PDR.0000084083.71422.16 matter of the brain occurs along the axons, this technique identifies white matter and indicates the direction of the fiber bundles within (9). In the absence of restriction, water diffusion is spatially uniform (i.e. isotropic). However, depending on the number, size, arrangement, and density of axons, as well as on the extent of myelination, water diffusion in the brain deviates from spherical and approaches the shape of an ellipsoid (i.e. anisotropic diffusion) with the long axis pointing along the axons [for a review, see Westin et al. (10)]. Therefore DTI provides information on the microstructure of white matter. To estimate the extent of anisotropy we calculated FA, which ranges in value between 0 and 1 for isotropic and onedimensional diffusion, respectively (11).

Several studies have used conventional MRI to investigate the immediate effects of brain injury in preterm newborns (12-14). Furthermore, on long-term follow-up conventional MRI was used to show that ex-preterm children have smaller 
brain volumes $(7,15)$, larger ventricles $(7,8,15)$, a smaller corpus callosum, and a reduced white matter volume (8).

DTI has been used to investigate the microstructure of white matter in preterms soon after birth. In one study Hüppi et al. (16) compared preterm children with or without white matter injury and found that several white matter areas, including the internal capsules, possessed a lower anisotropy in the preterm group with lesions. Furthermore, although anisotropy is directly correlated with gestational age (17), preterm children without white matter lesions possess a markedly lower anisotropy in several white matter locations when examined at a term equivalent time and compared with those born at term (18).

Therefore, it is clear that the microstructure of white matter is affected in newborn preterm children. A related and equally important question is whether these microstructural lesions would persist or affect the long-term development of white matter. Until now the long-term outcome of microstructural development of white matter has not been investigated by DTI.

We present a follow-up study on subjects recruited from the Stockholm Neonatal Project, which is a cohort of preterm births between 1988 and 1993 (6). The recruited subjects were selected on the basis of attentional deficits and hence represent a subgroup of the population of preterm children. We used DTI to compare a group of 11-y-old children, who had been born preterm (hereafter referred to as the preterm group) with a group of control children of the same age but born at term. The aim of this study was to investigate whether preterm birth leads to long-term disturbances in the microstructural integrity of white matter.

\section{METHODS}

Subjects. The children in the preterm group were selected from participants of the Stockholm Neonatal Project, a follow-up study of preterm births occurring between 1988 and $1993(6,19)$. There were a total of 182 children at $5 \mathrm{y}$ of age (19), who were subsequently followed up and available for selecting as our study group. Because of the narrow age span (9.67-12.17 y) we required and the other inclusion criteria (detailed below), we had a group of 10 children available (all of whom agreed to participate). In one child the DTI scan was not successful.

Inattentiveness and hyperactivity are common problems in preterm children (4-8), and the subjects were part of a functional MRI study in which the inclusion criteria were based on motor activity and distractibility scores. We used the NEPSY scale (20) and included children with rated motor-activity and distractibility scores, which correspond to more than 3 and more than $2 \mathrm{SD}$ above the population mean, respectively. In addition we excluded all subjects with evidence of PVL or IVH (as defined by the neonatal ultrasound examinations) or having an IQ $<80$ at 5 y of age.

The MRI images (collected in our study at $11 \mathrm{y}$ of age) of the children included in the study were examined by two pediatric neuroradiologists (as described below). Despite the negative neonatal ultrasound results, three children in the preterm group showed signs of mild PVL.
The control subjects were selected from a group of 125 healthy, term-born children who had been recruited to the follow-up study at 5 years of age (19). With this process seven of the nine preterm children had matched control subjects. The other three control subjects were recruited through advertising. The inclusion criteria for the control subjects were a gestational age of at least $37 \mathrm{wk}$ and a birth weight of at least $2501 \mathrm{~g}$, and they were matched to the preterm group for age and sex. We had successful DTI scans for nine children in the preterm group (three girls; birth weight, $1098.4 \pm 89.2 \mathrm{~g}$, gestational age, $28.6 \pm 1.05 \mathrm{wk}$ ) and in 10 control subjects (three girls). At the time of scanning the ages of the children in the preterm and control groups were $10.9 \pm 0.29$ and $10.8 \pm 0.33 \mathrm{y}$, respectively ( $p=0.83$, two-tailed $t$ test).

Ethical considerations. We obtained an informed consent from the parent of each participant. The Ethical Committee at the Karolinska Hospital in Stockholm, Sweden, approved the study.

MRI data acquisition. All subjects underwent a single scanning session on a 1.5-T General Electric Signa Echospeed (Milwaukee, WI, U.S.A.) scanner. The diffusion scheme was based on a set of directions, originally suggested by Jones et al. (21) and further improved by Skare et al. (22) by using 20 diffusion-weighted, noncollinear directions. We collected images in these 20 directions $\left(b=1000 \mathrm{~s} / \mathrm{mm}^{2}\right)$ as well as five nondiffusion-weighted images $\left(b=0 \mathrm{~s} / \mathrm{mm}^{2}\right)$.

We used a single-shot, diffusion-weighted, echo planar imaging sequence, triggered with pulse gating with a delay of 300 $\mathrm{ms}$. The inplane resolution was $1.72 \mathrm{~mm} \times 1.72 \mathrm{~mm}$. The slice thickness was $5 \mathrm{~mm}$ with no interslice spacing, and 18 horizontal slices were acquired from each subject.

Spin-echo $\mathrm{T}_{1}$-weighted images (TR/TE 500/8, three excitations) were also collected for each subject, including 18 axial slices with 5-mm slice thickness, no interslice spacing, and an inplane resolution of $1.72 \mathrm{~mm} \times 1.72 \mathrm{~mm}$.

Neuroradiologic examination. Two pediatric neuroradiologists independently examined the $\mathrm{T}_{1}$ - and $\mathrm{T}_{2}$-weighted images from each child. The $T_{2}$ images were the nondiffusionweighted images of the diffusion scheme. The radiologists were blinded to which group a child belonged. They used published criteria for diagnosis (23) and graded the severity of PVL on a scale from 0 to 3 , where $0=$ no; $1=$ mild; $2=$ moderate; and $3=$ severe. Each radiologist identified three children in the preterm group to have signs of PVL. They identified the same three children, and both gave each of the children a grade of 1 .

FA images. All volumes that contained slice(s) with signal dropout because of movement were excluded. The average number of images that had to be removed was 1.9 (maximum, 9 ). There was no group difference between the numbers of the excluded images ( $p=0.54$, two-tailed $t$ test).

We compensated for eddy current distortions and corrected for head-movement [i.e. movement that occurs between as opposed to during image acquisitions] (24) before estimating the diffusion tensor. From the tensor FA images were calculated (11).

To compare movement between the groups, for each subject we quantified movement as the mean of the absolute value of 
the rotations along each of the Cartesian axes. These values were obtained from the movement correction algorithm. We performed separate $t$ tests for each of the movement variables at a confidence level of 0.05 .

For spatial normalization (25) we used the mean of the five nondiffusion-weighted images for each subject and normalized it to a common template. The normalization parameters, which we obtained for each subject, were then applied to the FA image of the same subject.

Coherence images. For the present purpose we define coherence as the scalar measure that quantifies how well the main direction of water diffusion in a given voxel aligns with the main direction of water diffusion in all of its neighboring voxels (26). This measure is similar to the one put forth by Basser and Pierpaoli (11). For further details see the appendix to this article.

White matter probability maps. For each subject the mean of the five nondiffusion-weighted images were segmented with an automatic procedure as implemented in the SPM software (Wellcome Department of Cognitive Neurology, London, England). In the segmentation procedure each voxel was given probability values for possessing gray matter, white matter, or cerebrospinal fluid. The probability maps for the different tissue types were saved in separate images. In our analysis we only used the images of the white matter probability maps, but before the statistical comparison they were smoothed with a 5-mm Gaussian filter to obtain autocorrelation values similar to that of the FA images.

Statistical analysis of images. Group comparisons of the FA images were performed in SPM software with an absolute threshold of 0.15 (i.e. only considering voxels in which FA > 0.15 ). The rationale behind this threshold is 2-fold: first, small FA values, which normally indicate isotropic diffusion, are affected by noise and bias this measure to suggest anisotropy; and second, this threshold had been found to be reliable (27) to confine the analysis to white matter (i.e. FA values of white matter regions are larger than that of gray matter and cerebrospinal fluid).

As we did not have an a priori hypothesis for the outcome of the group comparisons, we performed a global comparison analysis (i.e. considering all the intracerebral voxels with $\mathrm{FA}>$ 0.15 , instead of a volume of interest), using a $t$ test and correcting for multiple comparisons $(28,29)$.

Analogous group analyses were also performed on the coherence images and the white matter probability maps. In these cases no threshold was applied.

Statistical analysis of brain size. The spatial normalization procedure, using the SPM software, results in 12 variables, which describe the linear transformations required to align an image to the template (30). Of the 12 variables, three describe the scaling along the three Cartesian axes. We extracted the values for scaling in the $x$ and $y$ directions from the results of the normalization procedures and compared them between the groups using $t$ test and a significance level of 0.05 . Scaling in the $z$ direction was not evaluated because we only obtained 18 slices for each subject and this did not provide full coverage of the brains.

Image and statistical analyses were performed using Matlab R12.1 (MathWorks Inc, Natick, MA, U.S.A.). When appropriate, the results and descriptive measures of the subjects are given as mean \pm SEM. The SPM software, which we used for all our movement corrections, statistical calculations, and normalization procedures, uses validated methods. A large number of references, detailed explanations, course notes, and an extensive mailbase can be found on the Internet (http://www.fil.ion.ucl.ac.uk/spm/).

\section{RESULTS}

Group comparison of FA and coherence. We first compared the FA images between the groups. This analysis resulted in three clusters (Table 1) in which the children in the preterm group had a significantly lower FA value compared with the control subjects. One of these clusters overlaps with the region of the posterior corpus callosum, and the other two are within the locality of the internal capsules bilaterally (Fig. 1).

We compared parameters of subject head movement that we obtained from the motion correction algorithm but found no group differences (Table 2). This indicated that our findings were not caused by image artifacts that could result from differences in the amount of movement between the groups.

The analysis of the coherence images, in which we compared the preterm and control groups, failed to indicate statistically significant differences between the groups.

Group comparison of white matter probability. The analysis for group differences resulted in several clusters in which the children in the preterm group had a lower probability of white matter. A search for voxels in which the children in the preterm group had both a lower FA value and a lower white matter probability resulted in 888 voxels. The three FA clusters together contained 2164 voxels (Table 1). Therefore $41 \%$ of these voxels had a lower probability for white matter in children of the preterm group. However, 821 of these 888 voxels were within the corpus callosum cluster (Fig. 2), and that cluster contains 1150 voxels. Thus $71 \%$ of the corpus callosum cluster in the FA analysis contained voxels in which the members of the preterm group had lower white matter probability whereas only $6 \%$ of the FA clusters in the internal capsules had this property.

Fiber directions in the internal capsule. In addition to comparing the FA images we also extracted the directional information within the DTI data. We displayed small lines indicating the $x$ and $y$ coordinates of the main direction of water diffusion within each voxel. In one case these directions were overlaid on the FA image to observe the relationship between anisotropy and fiber directions (Fig. 3A). This indicates that both the posterior and the anterior limbs of the internal capsule are equally anisotropic although the fiber directions are different. We also color-coded the $x, y$, and $z$

Table 1. Regions in which the children in the preterm group had lower FA values

\begin{tabular}{lccc}
\hline \multicolumn{1}{c}{ Significant clusters } & $\begin{array}{c}\text { Corrected } \\
p \text { value }\end{array}$ & $\begin{array}{c}\text { Cluster size } \\
\text { (voxels) }\end{array}$ & Location $(x, y, z)^{*}$ \\
\hline Posterior corpus callosum & $<0.001$ & 1150 & $(0,-46,15)$ \\
Left internal capsule & $<0.001$ & 320 & $(-15,-12,16)$ \\
Right internal capsule & $<0.001$ & 694 & $(10,-6,12)$ \\
\hline
\end{tabular}

* The coordinates refer to Talairach space in units of millimeters. 

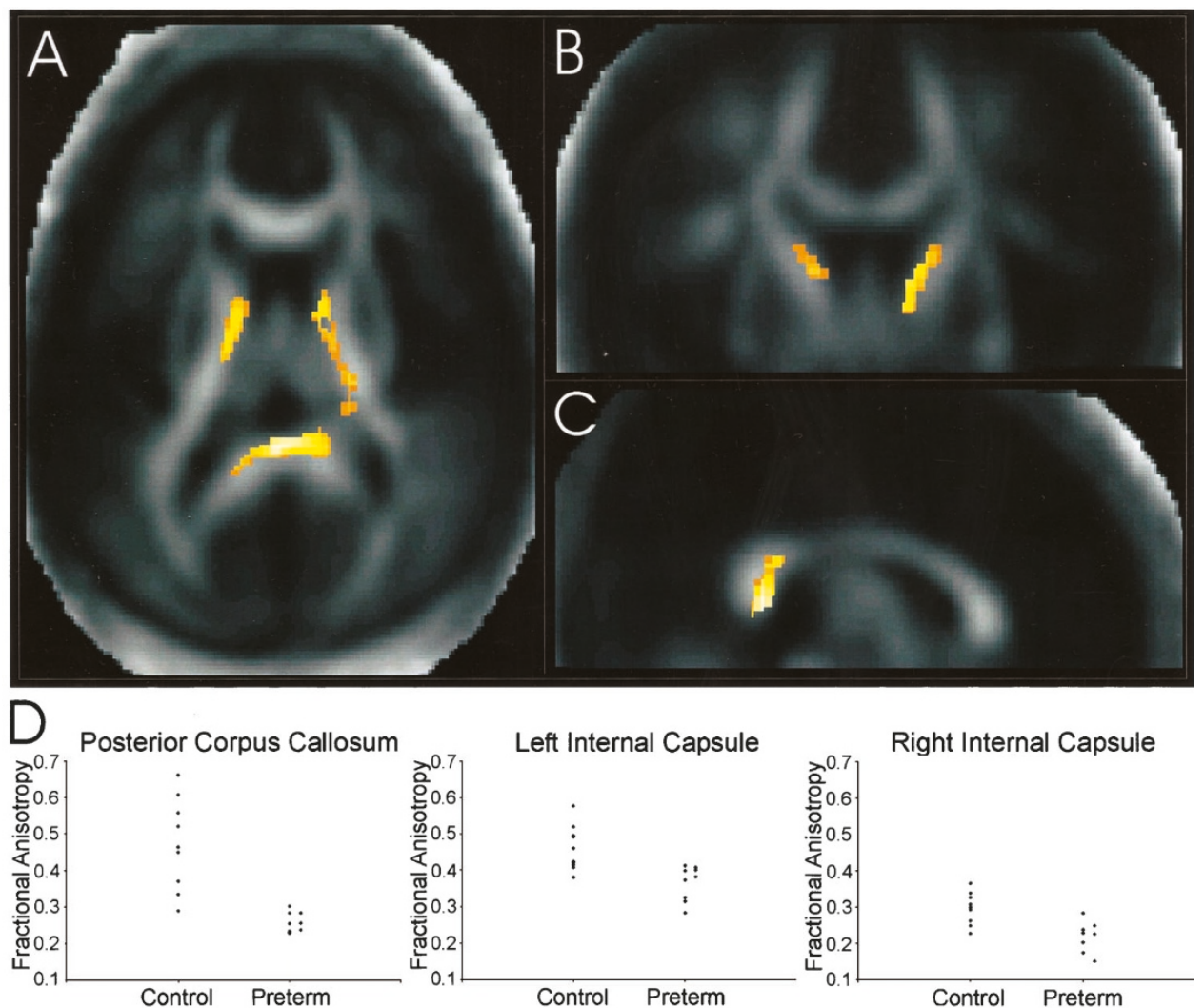

Figure 1. Significant clusters of group differences in FA values. $A$, horizontal section intersecting all three clusters (the posterior corpus callosum and the internal capsules bilaterally). $B$, coronal section intersecting the clusters in regions of the internal capsule. $C$, sagittal section intersecting the cluster within the posterior corpus callosum. $D$, plots of FA values from the peak voxel of the three clusters in $A-C$. The FA values corresponding to the children with mild PVL are displaced slightly to the right.

Table 2. Group comparison of head movement during scanning

\begin{tabular}{lllc}
\hline Rotations & \multicolumn{1}{c}{ Preterm } & \multicolumn{1}{c}{ Control } & $p$ value \\
\hline Pitch & $0.0071 \pm 0.0016$ & $0.0064 \pm 0.0011$ & 0.718 \\
Yaw & $0.0036 \pm 0.00067$ & $0.0040 \pm 0.00058$ & 0.643 \\
Roll & $0.0015 \pm 0.00012$ & $0.0015 \pm 0.00012$ & 0.667 \\
\hline
\end{tabular}

The figures represent the average rotational movement between the successful acquisitions of images. The quantities pitch, yaw, and roll have a unit of radians.

coordinates of the main direction and used this color plot as the background (Fig. 3B), which shows the relationship between the two-dimensional projection of the diffusion vector and its true, three-dimensional representation. These procedures indicated that the direction of white matter fiber bundles was different between the anterior and the posterior limbs of the internal capsules. The anterior limb contains fibers with axial orientation whereas the fibers in the posterior limb run in the superior-inferior direction.

Group comparison of brain size. During the normalization process the brains of the children in the preterm group had to be magnified significantly more than those of the control subjects. This difference was about $7 \%$ in the $x$ direction (left-right) and about $4 \%$ in the $y$ direction (anterior-posterior; Table 3). Thus the children in the preterm group tended to have smaller brains.

\section{DISCUSSION}

In this study we used DTI to show that preterm birth results in disturbances in the white matter microstructure that are still detectible at 11 y of age. In particular, white matter integrity of children in the preterm group was poorer in the posterior corpus callosum and within regions of both the anterior and posterior internal capsules bilaterally. We calculated a coherence measure, which suggested that the lower anisotropy in the preterm group is not likely to result from the disorganization of white matter fibers. Furthermore, we found indications that the children of the preterm group tended to have smaller brains.

Children were only included in the preterm group if they had not had ultrasound evidence of PVL or intracranial bleeding. Ultrasound examinations predict well the existence of IVH and PVL on autopsy (31-33), although false negatives do occur and another study indicates that a normal white matter echogenicity on ultrasound is not a good predictor of a normal MRI in preterm infants (34). For this reason the MRI images of each child were further examined for signs of PVL, and three members of the preterm group were diagnosed with a mild reduction of periventricular white matter volume. However, as

Table 3. Group comparison of brain size

\begin{tabular}{cccl}
\hline Scaling & Preterm & Control & $p$ value \\
\hline$x$ & $1.16 \pm 0.018$ & $1.08 \pm 0.01$ & $<0.001$ \\
$y$ & $1.05 \pm 0.014$ & $1.01 \pm 0.01$ & $<0.05$ \\
\hline
\end{tabular}




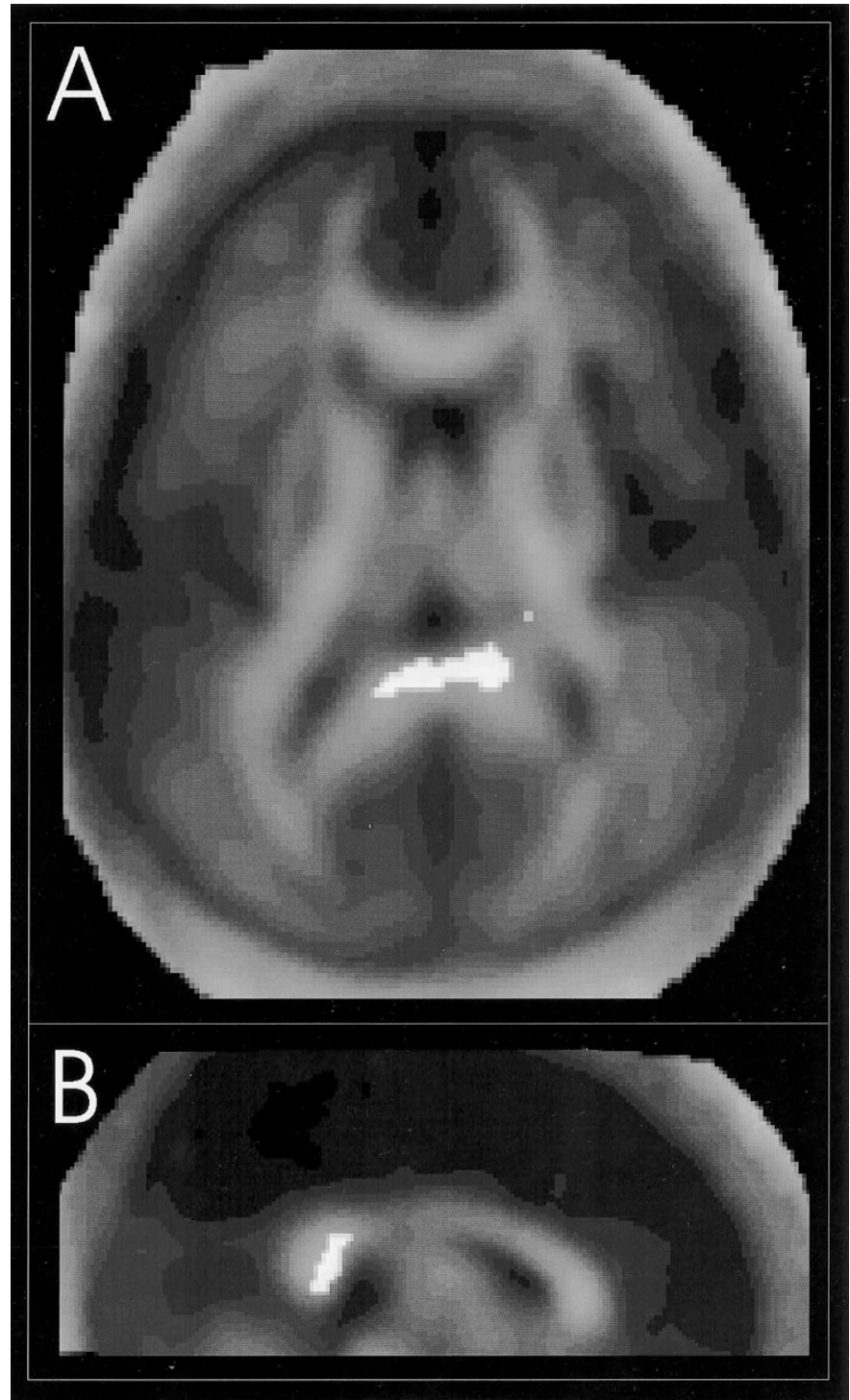

Figure 2. Significant group difference of white matter probability and FA. The cluster at the posterior corpus callosum in which the preterm group had both a lower FA value and white matter probability is displayed in horizontal $(A)$ and sagittal $(B)$ view in the same slices as Figure 1, $A$ and $C$, respectively. The clusters are overlaid on the average of the FA images of all the subjects.

the FA values of these three children were not outliers and they were distributed randomly about the mean of their group (Fig. $1 D$ ), we chose to allow them into the analyses. Furthermore, it is possible that more subjects would have shown signs of PVL had the $T_{1}$ - and $T_{2}$-weighted images been of higher quality. The fact that the three subjects with the most obvious sings of PVL were good representatives of the preterm group (at least on FA comparison) showed that the decrease in FA was not directly related to PVL. Therefore, it is not likely that the quality of the anatomic $\mathrm{T}_{1}$ - and $\mathrm{T}_{2}$-weighted images hindered our ability to draw our conclusions. Finally, our inclusion criteria required inattentiveness and hyperactivity. Although these symptoms are common in preterm children, our findings cannot be directly extended to preterm children without these symptoms.
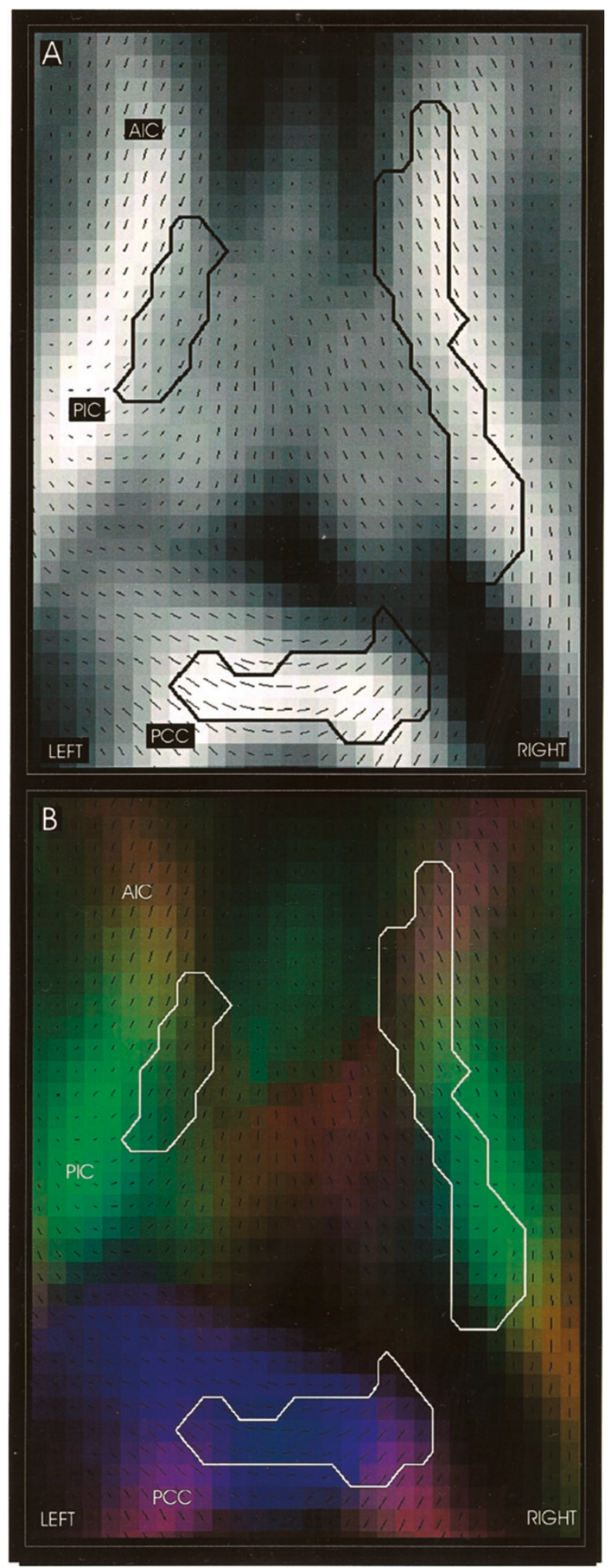

Figure 3. Horizontal projections of the principal directions of anisotropy. In each voxel the small line represents the horizontal projection of the largest eigenvector of the diffusion tensor. The length of the line is weighted by the anisotropy (FA value). The contour lines are outlines of the significant clusters from Figure 1. The vector plots are overlaid on the average of the FA images of all subjects $(A)$ or on a color-coded image where, blue, red, and green represent left-right, anterior-posterior, and superior-inferior directions, respectively, on the basis of the three components of the major direction of diffusion in a given voxel $(B)$. ( $P C C$, posterior corpus callosum; $P I C$, posterior internal capsule; $A I C$, anterior internal capsule; $A C C$, anterior corpus callosum) 
Main findings. In a recent study Hüppi et al. (16) compared preterm subjects with or without white matter injury. They made their comparison at term using DTI and found that anisotropy in the internal capsule was lower in those with white matter injury. In addition, it is possible to show by DTI that even in seemingly uncomplicated cases of preterm birth the developing brain takes a different course from those of fullterm children (18). It seems that during the period between birth and term, the development of fiber organization or myelination of preterm children does not keep up with the intrauterine development of infants born at term. Until now, however, it was not clear whether these initial alterations within the internal capsules would be detectible, using DTI, on a longterm follow-up. The results of our study show that the microstructural development of the internal capsule is affected throughout the maturation process, at least up to $11 \mathrm{y}$ of age.

The unfavorable effects of preterm birth have been implicated also in the hindered development of the posterior corpus callosum. Previously, Stewart et al. (8) found that ex-preterm children had a thinner corpus callosum at $15 \mathrm{y}$ of age. Peterson et al. (7) reported a similar finding in a group of 8-y-olds. However, to date these findings have not been supported with the DTI method. In this study we found that the posterior corpus callosum had a lower FA in the preterm group, indicating the possibility of smaller fiber bundles in this region.

Coherence. It has been suggested previously that the lower FA in preterm children is attributable to the fact that their fibers are not as well organized (16). To investigate this idea further, we calculated a coherence measure $(11,26)$. This measure indicates how well the main fiber directions align in neighboring voxels (see the appendix to this article). Based on our analysis, we suggest that the lower FA values in preterm children (at least on long-term follow-up) are not likely to result from the disorganization of axonal pathways. Instead, the decreased anisotropy is likely to be related to one or the combination of 1) reduced axon diameter, 2) fewer axons, and 3) poorer myelination.

Differences in white matter volume. The results of the FA analysis may be attributable to a volumetric difference in white matter between the two groups, which was not corrected for by the spatial normalization process. Hence we performed a group analysis on the white matter probability maps. This analysis showed that the children in the preterm group had a significantly lower white matter probability within the FA cluster at the posterior corpus callosum (Fig. 2). This result was not unexpected as preterm birth had been associated with thinning of the posterior corpus callosum $(7,8)$ and enlargement of lateral ventricles $(7,13$, $14,35)$. The lower white matter volume in the preterm group may thus represent a confounder for the group difference in FA values in the posterior corpus callosum. The FA difference in the internal capsules, however, could not be explained by white matter volume differences between the groups.

Reduced brain size. We also found indications of reduced brain size for members of the preterm group. It turns out that during the normalization procedure (30), the brains of children in the preterm group needed to be scaled more than the brains of the term children by approximately $7 \%$ in the left-right and approximately $4 \%$ in the anterior-posterior direction. Our find- ing is in agreement with previous reports on reduced brain volumes in older children and adolescents with preterm birth $(7,15)$. In particular, in the study by Peterson et al. (7), cortical volumes of ex-preterm children were smaller in the sensorimotor and premotor cortical regions as well as in the basal ganglia and the corpus callosum. Also, Inder et al. (14) found that preterm children with PVL had reduced gray and myelinated white matter volumes compared with term children without PVL. It is therefore reasonable to think that the internal capsules and the corpus callosum may be less anisotropic in preterm children because they consist of fewer fibers connecting smaller cortical or subcortical regions.

Axonal directions in the internal capsules. The internal capsules are conventionally divided into the anterior and posterior limbs. Histologic studies have shown that in the anterior limb the fibers run in the horizontal plane whereas in the posterior limb they have a superior-inferior (corticospinal) directionality (12). Our results confirm this finding (Fig. 3). Furthermore, the significant clusters in which we found lower FA values for the preterm group involve axons with both horizontal and superior-inferior projections. This finding indicates that the white matter injury in preterm children is specific for the location of the internal capsules rather than for the particular axonal pathways involved (as would happen in anterograde white matter degeneration for example).

\section{CONCLUSIONS}

In this study we presented the novel finding that preterm birth (at least in children with attention deficits) is associated with a regionally specific white matter disturbance that is detectable using DTI at $11 \mathrm{y}$ of age. The affected areas are within the region of the internal capsules bilaterally and the posterior corpus callosum, although the latter one may be confounded by smaller white matter volumes in the preterm group. The reduced FA values in preterm children are more likely to be related to an impairment of myelination, a hindrance of axon diameter growth, a reduction in the number of axons, or a combination of these factors than to a significant reduction in the extent of organization (i.e. coherence) of white matter fiber tracks. The functional implications of this developmental disruption in the microstructure of white matter are not well known. The results of this study motivate future work to identify the possibly affected cognitive, sensory, or motor abilities.

Acknowledgment. The authors thank Dr. Miriam KatzSalamon for her assistance in the recruitment of patients.

\section{APPENDIX}

In principle the direction of water diffusion depends on the following factors: 1) extent of myelination, 2) axon diameter, 3) number of axons, and 4) the extent of organization (i.e. coherence) of fibers [reviewed by Westin et al. (10)]. Each of these factors is directly related to the anisotropy of water diffusion. Our aim with the coherence analysis was to eliminate one of the possible interpretations of FA (11). In practice, for each voxel, we averaged the absolute value of the dot product between the largest eigenvector of that voxel and the largest eigenvector of each of the neighboring voxels in three dimen- 


\section{Low Coherence}
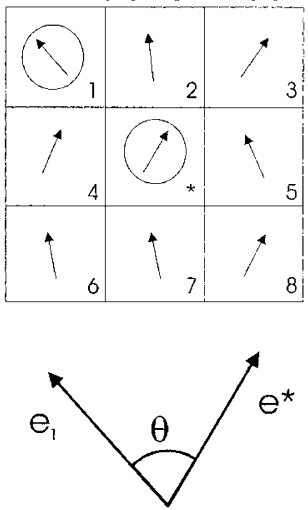

High Coherence

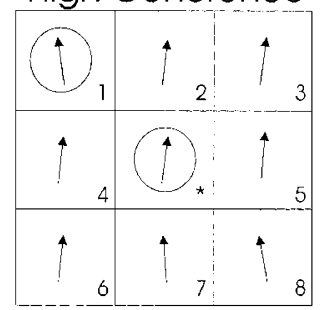

e

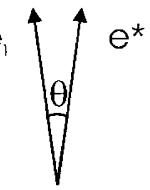

Figure 4. Explanation for the concept of coherence in the two-dimensional case Consider the two $3 \times 3$ grids as a representation of neighboring voxels within a plane in the brain. The arrows within each voxel represent the main direction of water diffusion in that voxel. The voxels in the grid on the left contain arrows that are more disorganized (i.e. less coherent) then the arrows within the grid on the right. The coherence index quantifies how well the arrows align. As the arrows represent the direction of water diffusion in a given voxel and water tends to diffuse along white matter fibers, this coherence measure in effect measures how well white matter fibers are organized in the brain. Analytically, one would measure the smallest positive angle between the main direction of water diffusion in a given voxel (here denoted by an asterisk) and the main direction of water diffusion in each of its neighboring voxels (numbered from 1 to 8 ), take the absolute value of the cosine of this angle, and average for the eight different combinations. This procedure is repeated for all the voxels in the brain. Take for example the center voxel and the top left voxel in each grid (magnified at the bottom, below each grid). It is clear that the angle between the arrows is larger in the grid with more-disorganized fibers. As the cosine of an angle runs between 0 and 1, this measure gives a numerical value to the degree of organization.

sions (for a pictorial explanation see Figure 4). From the definition of the dot product between two vectors we have:

$$
D_{\text {ave }}^{*}=\frac{\sum_{i=1}^{n} e^{*} \cdot e_{i}}{n}=\frac{\sum_{i=1}^{n}\left|e^{*} \| e_{i}\right| \cos \left(\theta_{i}\right)}{n}
$$

where $D_{\text {ave }}^{*}$ is the average dot product for a given voxel (denoted by $*), e^{*}$ is the unit vector indicating the principal direction of water diffusion in that voxel, $e_{i}$ is the unit vector indicating the principal direction of water diffusion in a voxel that is an immediate neighbor of the voxel denoted by $*, \theta_{i}$ is the smallest positive angle between $e^{*}$ and $e_{i}, n$ is the number of neighbors of the voxel denoted by *, and $\left|e^{*}\right|,\left|e_{i}\right|$ denote the length of the vectors $e^{*}$ and $e_{i}$.

If the vectors have a length of unity (which $e^{*}$ and $e_{i}$ do), then the dot product of two vectors is equivalent to the cosine of the angle between them. Furthermore, in three dimensions any given voxel has 26 immediate, contiguous neighbors. Hence the general expression above simplifies to:

$$
D_{\text {ave }}^{*}=\frac{\sum_{i=1}^{26} \cos \left(\theta_{i}\right)}{26}
$$

This measure ranges in value from 0 , when all the neighboring voxels have perpendicular eigenvectors, to 1 , when the eigen- vectors of all the neighboring voxels are aligned. Coherence is a scalar measure, similar to FA. The coherence images were normalized using the same parameters as that for the FA images (see FA images above in "Methods" section).

\section{REFERENCES}

1. Wood NS, Marlow N, Costeloe K, Gibson AT, Wilkinson AR 2000 Neurologic and developmental disability after extremely preterm birth. EPICure Study Group. N Engl J Med 343:378-384

2. Volpe JJ 2001 Neurology of the Newborn, 4th ed. WB Saunders, Philadelphia

3. Perlman JM 1998 White matter injury in the preterm infant: an important determination of abnormal neurodevelopment outcome. Early Hum Dev 53:99-120

4. Hack M, Flannery DJ, Schluchter M, Cartar L, Borawski E, Klein N 2002 Outcomes in young adulthood for very-low-birth-weight infants. N Engl J Med 346:149-157

5. Escobar GJ, Littenberg B, Petitti DB 1991 Outcome among surviving very low birthweight infants: a meta-analysis. Arch Dis Child 66:204-211

6. Katz-Salamon M, Forssberg H, Lagercrantz H 1997 The Stockholm Neonatal Project: very low birthweight infants in the late 20th century in Stockholm. Acta Paediatr 86 (suppl 419):1-43

7. Peterson BS, Vohr B, Staib LH, Cannistraci CJ, Dolberg A, Schneider KC, Katz KH, Westerveld M, Sparrow S, Anderson AW, Duncan CC, Makuch RW, Gore JC, Ment LR 2000 Regional brain volume abnormalities and long-term cognitive outcome in preterm infants. JAMA 284:1939-1947

8. Stewart AL, Rifkin L, Amess PN, Kirkbride V, Townsend JP, Miller DH, Lewis SW, Kingsley DP, Moseley IF, Foster O, Murray RM 1999 Brain structure and neurocognitive and behavioural function in adolescents who were born very preterm. Lancet 353:1653-1657

9. Moseley ME, Cohen Y, Kucharczyk J, Mintorovitch J, Asgari HS, Wendland MF, Tsuruda J, Norman D 1990 Diffusion-weighted MR imaging of anisotropic water diffusion in cat central nervous system. Radiology 176:439-445

10. Westin CF, Maier SE, Mamata H, Nabavi A, Jolesz FA, Kikinis R 2002 Processing and visualization for diffusion tensor MRI. Med Image Anal 6:93-108

11. Basser PJ, Pierpaoli C 1996 Microstructural and physiological features of tissues elucidated by quantitative-diffusion-tensor MRI. J Magn Reson B 111:209-219

12. Felderhoff-Mueser U, Rutherford MA, Squier WV, Cox P, Maalouf EF, Counsell SJ, Bydder GM, Edwards AD 1999 Relationship between MR imaging and histopathologic findings of the brain in extremely sick preterm infants. AJNR Am J Neuroradiol 20:1349-1357

13. Maalouf EF, Duggan PJ, Rutherford MA, Counsell SJ, Fletcher AM, Battin M, Cowan F, Edwards AD 1999 Magnetic resonance imaging of the brain in a cohort of extremely preterm infants. J Pediatr 135:351-357

14. Inder TE, Huppi PS, Warfield S, Kikinis R, Zientara GP, Barnes PD, Jolesz F, Volpe JJ 1999 Periventricular white matter injury in the premature infant is followed by reduced cerebral cortical gray matter volume at term. Ann Neurol 46:755-760

15. Nosarti C, Al Asady MH, Frangou S, Stewart AL, Rifkin L, Murray RM 2002 Adolescents who were born very preterm have decreased brain volumes. Brain $125: 1616-1623$

16. Hüppi PS, Murphy B, Maier SE, Zientara GP, Inder TE, Barnes PD, Kikinis R, Jolesz FA, Volpe JJ 2001 Microstructural brain development after perinatal cerebral white matter injury assessed by diffusion tensor magnetic resonance imaging. Pediatrics 107:455-460

17. Neil JJ, Shiran SI, McKinstry RC, Schefft GL, Snyder AZ, Almli CR, Akbudak E, Aronovitz JA, Miller JP, Lee BC, Conturo TE 1998 Normal brain in human newborns: apparent diffusion coefficient and diffusion anisotropy measured by using diffusion tensor MR imaging. Radiology 209:57-66

18. Huppi PS, Maier SE, Peled S, Zientara GP, Barnes PD, Jolesz FA, Volpe JJ 1998 Microstructural development of human newborn cerebral white matter assessed in vivo by diffusion tensor magnetic resonance imaging. Pediatr Res 44:584-590

19. Bohm B, Katz-Salamon M, Institute K, Smedler AC, Lagercrantz H, Forssberg H 2002 Developmental risks and protective factors for influencing cognitive outcome at $51 / 2$ years of age in very-low-birthweight children. Dev Med Child Neurol 44:508-516

20. Korkman M 1990 Manual NEPSY-Neuropsychological Assessment: 4-7 years (Swedish version). Psykologiförlaget AB, Stockholm

21. Jones DK, Horsfield MA, Simmons A 1999 Optimal strategies for measuring diffusion in anisotropic systems by magnetic resonance imaging. Magn Reson Med 42:515-525

22. Skare S, Hedehus M, Moseley ME, Li TQ 2000 Condition number as a measure of noise performance of diffusion tensor data acquisition schemes with MRI. J Magn Reson 147:340-352

23. Flodmark O, Lupton B, Li D, Stimac GK, Roland EH, Hill A, Whitfield MF, Norman MG 1989 Magnetic resonance imaging of periventricular leukomalacia (PVL) in childhood. AJNR Am J Neuroradiol 10:111-118

24. Andersson JL, Skare S 2002 A model-based method for retrospective correction of geometric distortions in diffusion-weighted EPI. Neuroimage 16:177-199

25. Ashburner J, Friston KJ 1999 Nonlinear spatial normalization using basis functions. Hum Brain Mapp 7:254-266

26. Klingberg T, Hedehus M, Temple E, Salz T, Gabrieli JD, Moseley ME, Poldrack RA 2000 Microstructure of temporo-parietal white matter as a basis for reading ability: evidence from diffusion tensor magnetic resonance imaging. Neuron 25:493-500

27. Jones DK, Simmons A, Williams SC, Horsfield MA 1999 Non-invasive assessment of axonal fiber connectivity in the human brain via diffusion tensor MRI. Magn Reson Med 42:37-41

28. Friston KJ, Holmes AP, Worsley KJ, Poline JP, Frith CD, Frackowiak RSJ 1995 Statistical parametric maps in functional imaging: a general linear approach. Hum Brain Mapp 2:189-210 
29. Friston KJ, Worsley KJ, Frackowiak RSJ, Mazziotta JC, Evans AC 1994 Assessing the significance of focal activations using their spatial extent. Hum Brain Mapp $1: 214-220$

30. Friston KJ, Ashburner J, Poline JB, Frith CD, Heather JD, Frackowiak RSJ 1995 Spatial registration and normalization of images. Hum Brain Mapp 2:165-189

31. Hill A, Melson GL, Clark HB, Volpe JJ 1982 Hemorrhagic periventricular leukomalacia: diagnosis by real time ultrasound and correlation with autopsy findings. Pediatrics 69:282-284

32. Fawer CL, Calame A, Perentes E, Anderegg A 1985 Periventricular leukomalacia: a correlation study between real-time ultrasound and autopsy findings: periventricular leukomalacia in the neonate. Neuroradiology 27:292-300

33. Nwaesei CG, Pape KE, Martin DJ, Becker LE, Fitz CR 1984 Periventricular infarction diagnosed by ultrasound: a postmortem correlation. J Pediatr 105:106-110

34. Maalouf EF, Duggan PJ, Counsell SJ, Rutherford MA, Cowan F, Azzopardi D, Edwards AD 2001 Comparison of findings on cranial ultrasound and magnetic resonance imaging in preterm infants. Pediatrics 107:719-727

35. Battin MR, Maalouf EF, Counsell SJ, Herlihy AH, Rutherford MA, Azzopardi D, Edwards AD 1998 Magnetic resonance imaging of the brain in very preterm infants: visualization of the germinal matrix, early myelination, and cortical folding. Pediatrics 101:957-962 